\title{
Increasing the Efficiency of Storage of Cryoproduct through the Introduction of Technical Solutions
}

\author{
S V Zaika ${ }^{1, a}$, Aksenova D.K. ${ }^{1}$, E A Shatokhin ${ }^{1}$ and S S Zaika ${ }^{1}$ \\ ${ }^{1}$ Samara University, Department Of Heat Engineering And Heat Engines, 443086 Moskovskoye shosse, 34, Samara, \\ Russia
}

\begin{abstract}
In various systems of aerospace engineering, transport, as well as in energy complexes for various purposes, cryogenic liquids are now widely used. Liquefied natural gas (LNG) is an environmentally friendly energy source that has several advantages over petroleum products. LNG using reduces the weight and size characteristics of tanks for storage and transportation. Reducing the loss of cryoproducts using low-potential power machines to ensure efficient storage of cryoproducts. Significant problems associated with the loss of its low-potential energy (caused by heat from the environment). There is a need to improve solutions to compensate for losses and expenses. This study examined the possibility of reducing the loss of cryoproducts when stored in tanks and transportation systems. Details were studied: assessment of losses of the cryoproduct and its capabilities for low-temperature energy, calculation of the loss compensation loop, calculation of the contour for generating additional energy based on a heat power plant, analysis of low-potential power plants, compensating losses of cryoproducts during storage. The main results of the research are the proposal to organize the LNG Storage, calculate the loss compensation loop and the supplementary energy circuit.
\end{abstract}

\section{Introduction}

The use of LNG in many branches of the agro-industrial complex as an energy carrier has obvious advantages due to a number of features, and first of all, its wide application possibilities, relatively low cost, environmental cleanliness, the possibility and convenience of transportation [1].

LNG as an energy carrier and natural gas derived from it can be used for:

- heat supply (production of steam and hot water) in boiler installations of residential and public buildings, as well as consumer services. The range of these units is quite wide and depends on the required heat output;

- heating of premises and supply of the population with hot water.

- power supply to remote deep areas by creating mobile gas turbine power stations capable of operating on gaseous fuels. When operating a gas turbine power plant, the heat of the exhaust gases can be used for the heating of nearby residential areas. The subject of the study is the thermophysical processes occurring during the storage of cryoproduct (LNG), and the object of investigation is a lowpotential power plant [2].

\footnotetext{
${ }^{\mathrm{a}}$ Corresponding author : zaika@ssau.ru
} 


\section{Estimation of storage losses}

When storing a cryoproduct, its losses are due to the low efficiency of thermal insulation and the heat input from the environment. It is possible to calculate the loss of the low-temperature heat of the cryoproduct lost during its storage by the following equation:

$$
\begin{gathered}
\Delta m=m_{\Sigma} \cdot \frac{\theta}{100} \\
Q_{\text {evap }}=\Delta m \cdot r+c_{p} \cdot \Delta m \cdot\left(T_{c}-T_{0}\right)
\end{gathered}
$$

where $\Delta m$ - mass of evaporated cryoproduct per day, $m_{\Sigma}$ - mass of stored cryoproduct; $\Theta$ - loss of product due evaporation, $\% Q_{\text {evap }}$ - low-potential heat of the evaporated cryoproduct; $r$ - heat of evaporation of cryoproducts; $T_{c}$ - cryoproduct temperature; $T_{o}$ - ambient temperature.

Cryoproduct has a high specific low-temperature energy potential, which can be used as a "refrigerator" in a thermal machine operating along the Curzon-Alborne cycle. The loss of disposable energy $L$ per day, provided that the low-potential heat of the cryoproduct is used according to the Curzon-Alborne cycle, is calculated as follows:

$$
\begin{gathered}
L=\frac{Q \cdot \eta}{1-\eta} \\
\eta=1-\sqrt{\frac{T_{2}}{T_{1}}}
\end{gathered}
$$

Losses from evaporation in tanks, depending on its dimensions and designs. When choosing the insulation, the required liquid used and the fact that the thickness of the insulation increases with an increase in the volume of the vessels more slowly than the diameter of the vessel. When choosing the isolation of tanks, economic factors should be considered. The use of vacuum-multi-layer insulation allows a 10-fold reduction in oxygen loss compared with vacuum-powder insulation. However, the difference in their cost is so great that the payback is calculated in tens of years.

Specific volumetric daily losses of low-grade heat of the cryoproduct depending on the volume of the reservoir and specific volumetric daily losses of available energy under the condition of using lowpotential heat along the Curzon-Alborn cycle are determined by the equation:

$$
\begin{gathered}
q_{V}=Q / V \\
l_{V}=L / V
\end{gathered}
$$

where $\mathrm{q}_{\mathrm{v}}$ - specific daily volumetric losses of low-grade heat of the cryoproduct; $\mathrm{l}_{\mathrm{V}}$ - the specific daily volume losses of the available energy, provided low-potential heat is used in the Carnot cycle; V - tank volume.

Even though the relative losses of the cryoprotectant decrease with the increase in the volume of the reservoir, the absolute values of losses of low-grade heat increase, which is quite logical.

The calculation results show a significant amount of the low-potential energy of the cryoproduct lost during storage. LNG has the greatest potential of cryogenic energy that lost during storage. Based on these results, the problem arises of determining the ways of solving the reduction in mass losses of the cryoproduct due to evaporation in tanks.

Calculation of specific inflows per running meter of pipelines of various diameters. The calculated values of specific heat inflows are presented in Table 1 and in Figure 1. 
Table 1. Values of specific inflows per running meter of pipelines

\begin{tabular}{cccc}
\hline $\begin{array}{c}\text { Diameter } \\
\text { of the pipeline }\end{array}$ & $\begin{array}{c}\text { Size of inner } \\
\text { tube, DxS }\end{array}$ & $\begin{array}{c}\text { Casing diameter, } \\
\mathbf{D x S}\end{array}$ & $\begin{array}{c}\text { Specific } \\
\text { heat input, [W / m] }\end{array}$ \\
\hline 15 & $18 \times 1,4$ & $100 \times 2$ & 0,9 \\
25 & $28 \times 1,4$ & $100 \times 2$ & 1,1 \\
50 & $56 \times 2$ & $150 \times 2,5$ & 1,2 \\
65 & $70 \times 2$ & $150 \times 2,5$ & 1,3 \\
100 & $100 \times 2$ & $200 \times 2,5$ & 1,5 \\
150 & $150 \times 2,5$ & $250 \times 3$ & 2,2 \\
200 & $200 \times 2,5$ & $300 \times 3$ & 3,0 \\
250 & $250 \times 3$ & $350 \times 3,5$ & 4,0 \\
300 & $300 \times 3$ & $408 \times 4$ & 5,2 \\
400 & $408 \times 4$ & $500 \times 4$ & 8,0 \\
\hline
\end{tabular}

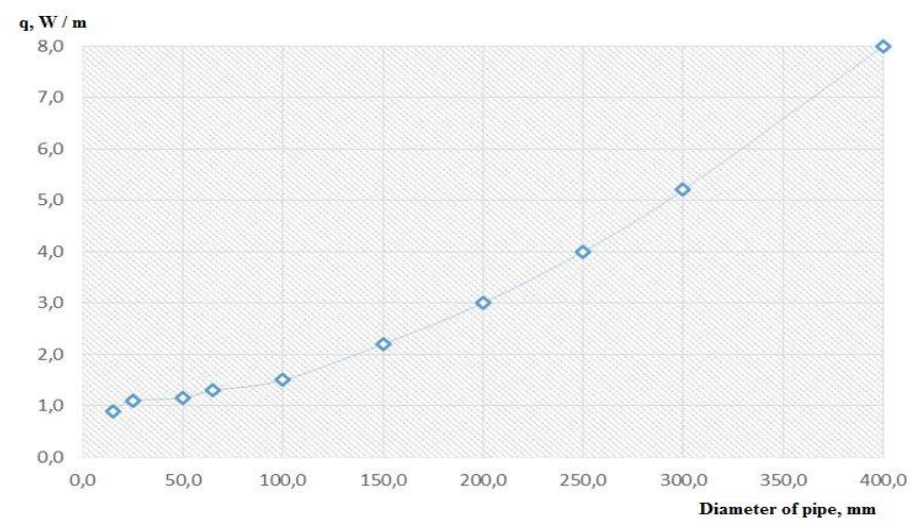

Figure 1. Dependence of specific heat input per 1 meter of pipeline from its internal diameter

Figure 2 shows the scheme for transporting a cryogenic liquid over distances. In such a system, the heat supplied to the cryoproduct in the pump is given to a low-pressure liquid in the heat exchanger, and the injected liquid is already supplied to the pipeline even at a pressure (greater than the saturation pressure). The problem was considered under the condition that the liquid reaches the gate in a saturated state. Numerous examples have been calculated for various flow rates, pump discharge pressures, pipe diameters and pipeline lengths.

\section{Options for organizing the LNG storage facility}

Three different options for organizing LNG storage were selected. The first combination consists of 4 tanks with a capacity of $112 \mathrm{~m}^{3}$, the second - from 2 tanks with a capacity of $248 \mathrm{~m}^{3}$, the third - from 8 tanks with a capacity of $49.50 \mathrm{~m}^{3}$. The logic of the organization consists of selecting a more advantageous option, based on the considerations of the least loss of evaporation while the cylinders are empty. The number of tanks of certain volumes was selected on the basis of the mass of fuel required to operate the gas piston plant (GPU) with a capacity of $1 \mathrm{MW}$ for 28 days, which is 168480 $\mathrm{kg}$. LNG preparation and storage systems are shown in Figure 2. 


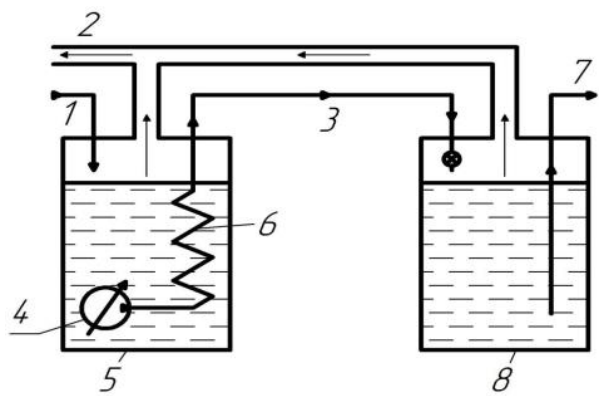

Figure 2. Liquefied gas transmission system

1- liquid intake; 2 - return or release of steam;3 - pipeline; 4 - the pump; 5 - pump reservoir;

6-heat exchanger; 7 -to the consumer of the liquid; 8 - remote storage

During operation, tanks are used alternately. Losses in the mass of the cryoproduct in the used tank are not considered, while in the remaining tanks losses occur while they are in the standby mode. The amount of loss depends on the selected storage scheme and the characteristics of the tanks involved (Table 2).

Considering the processes of heat transfer, the efficiency in the Curzon-Alborne cycle is calculated as follows:

$$
\eta^{\prime}=1-\left(T_{2} / T_{1}\right)^{1 / 2}
$$

where $T_{2}$ - cold source temperature, $\mathrm{K} ; T_{1}$ - temperature of the heat reservoir, $\mathrm{K}$.

With an increase in the maximum permissible temperature of fuel elements, the average temperature of the working fluid $\mathrm{T}$ 'and the efficiency increase. At the same time, the average temperature of the working fluid increases slowly, and the efficiency is fast enough. efficiency can be improved by regeneratively heating the working fluid to a high temperature. When you receive even a small amount of heat from the environment, the liquid (LNG) partially passes into the gaseous form, this leads to gas leaks. The amount of evaporated liquefied natural gas (evaporation loss) is rapidly becoming significant, their energy value can not be neglected. Therefore, in general, several solutions are proposed to reduce the losses of the liquid phase of the cryoproduct, based on the use of a heatnegative power plant having a loss compensation contour.

Table 2. Options for organizing the LNG storage facility

\begin{tabular}{|c|c|c|c|}
\hline The scheme & a & b & c \\
\hline $\begin{array}{c}\text { Number of } \\
\text { tanks }\end{array}$ & 2 & 4 & 8 \\
\hline $\mathbf{V}, \mathbf{~ m}^{\mathbf{3}}$ & 248 & 112 & 49,50 \\
\hline $\mathbf{M}, \mathbf{k g}$ & 84 & 42 & 21060 \\
\hline Loss per day, \% & 240 & 212 & 0,12 \\
\hline $\begin{array}{c}\text { Losses in mass in one } \\
\text { tank per day, kg }\end{array}$ & 75,09 & 0,12 & 25,272 \\
\hline $\begin{array}{c}\text { Losses for all time in } \\
\text { all tanks, kg }\end{array}$ & 16 & 50,6 & 2476,66 \\
\hline
\end{tabular}

\section{Possible technical solutions for loss reduction cryoproduct at its storage}

Technical solutions are proposed for reducing the loss of cryoproducts during storage by using a plant that consists of two circuits, one of which is a loss compensation contour and the other is a contour for 
generating additional energy. The circuit for generating additional energy can be realized based on the Stirling engine.

The loss compensation contour includes:

- cryogenic tank with liquefied natural gas;

- throttle valve. This is a special valve used in refrigeration units and heat pumps in which a process of throttling occurs, that is, an irreversible expansion of the liquid with a decrease in pressure and temperature.

- a gas receiver intended for the accumulation of an evaporating liquid phase;

- heat exchanger - evaporator, which is necessary to cool the gas heated after the compressor with liquid methane to the temperature at which it will be completely in the liquid state;

-centrifugal compressor [3].

The gas from the suction chamber located near the axis of rotation is sucked into the impeller, enters its interblade channels and is pushed through these channels by the centrifugal forces to exit the impeller. With this movement, a constantly increasing centrifugal force acts on the gas, that increase gas velocity. From the thermodynamics of the flow, we know that when the gas pressure flow is accelerated, it decreases. The blade channels of the impeller have an expanding shape, and this leads to an increase in pressure.

The total effect of these two influences leads to an increase in pressure, so that at the exit of the impeller the absolute gas velocity becomes maximum and its pressure rises. Then the gas enters the fixed vane diffuser, where its braking takes place with a corresponding increase in pressure. Then, the flow in the reverse guiding device passes through the interscapular channels with increasing cross section and enters the next stage.

The principle of operation of the loss compensation circuit during evaporation is as follows. Liquefied natural gas is charged into the tank with a capacity in accordance with the selected scheme of the storage park at a temperature of $138 \mathrm{~K}$ and a pressure of $0.6 \mathrm{MPa}$. The evaporated natural gas flows through the drain valve to the receiver. Accumulated losses per day are compressed by a centrifugal compressor up to $1 \mathrm{MPa}$.

The operating time of the compressor is 20 minutes. The drive of the centrifugal compressor is an electric motor that operates on the energy generated by the Stirling engine, depending on the chosen circuit. After the compressor, the heated gas is cooled by liquid methane in the heat exchangerevaporator and then throttled to $0.65 \mathrm{MPa}$. Liquefied gas returns to the tank.

\section{Scheme of plant based on the Stirling engine}

The drive of the centrifugal compressor can be an electric motor running on the energy generated by the Stirling engine. After the compressor, the heated gas is cooled by liquid methane in the heat exchanger-evaporator and then throttled to 4.5 MPa. Liquefied gas returns to the tank.

The second circuit of the heat-negative installation based on the Stirling engine includes:

- Stirling engine, which includes three heat exchangers: a heat exchanger for the expander cavity, a compressor cavity, a regenerative heat exchanger. In Stirling engines, the heat exchanger of the expander cavity, perceiving heat from an external source at a high temperature, transfers it to the engine's working body located in the working cavity adjacent to the expansion cavity [4].

The principle of operation of the circuit based on the Stirling engine is as follows (Figure 3). The compressor cavity of the Stirling engine is cooled by liquefied natural gas to a temperature of $158 \mathrm{~K}$, and the expander cavity is heated from the exhaust gases of the gas piston unit to a temperature of 400 $\mathrm{K}$. The actual engine power is $4.8 \mathrm{~kW}$. Liquefied gas, passing through the heat exchanger of the compressor cavity of the Stirling engine, enters the heat exchanger-utilizer, where it is heated by exhaust gases from the GPU. After full gasification of gas, it is disposed of in the GPU. 


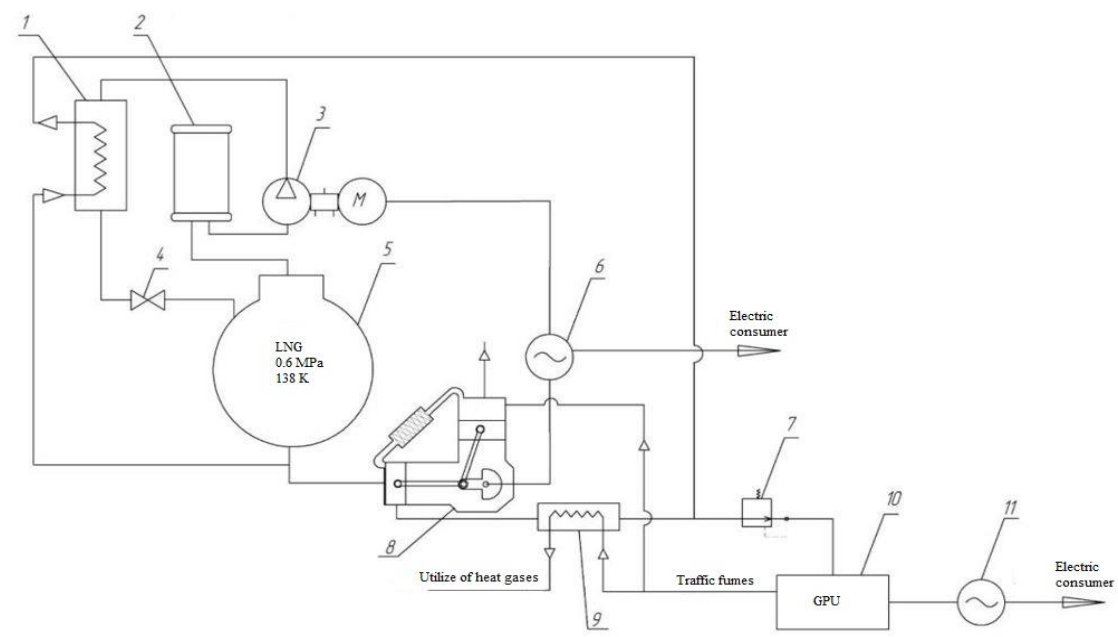

Figure 3. Scheme of installation based on the Stirling engine

1 - heat exchanger-evaporator; 2 - Gas receiver; 3-compressor; 4 - throttle valve; 5 - LNG tank; 6 - electric generator; 7 - reducer; 8 - Stirling engine; 9 - heat exchanger-utilizer; 10 - GPU; 11 - power generator.

The advantage of the proposed low-temperature power plant based on the Stirling engine:

- high energy efficiency;

- proven technology of small-scale production of engines.

Disadvantages of the proposed low-temperature power plant based on the Stirling engine:

- the difficulty of ensuring the tightness of the Stirling engine;

- The complexity of the design of the Stirling engine [5].

\section{Low-potential energy converters}

It is proposed to use thermoelectric generator modules (TGM) to realize the low-temperature potential of LNG, which are now widely used. The power generated by such generators ranges from a few milliwatts to several kilowatts and is ultimately determined by the economic feasibility of choosing this method of energy conversion [6].

At present, the line of modern thermoelectric generators provides the possibility of obtaining electric power with an efficiency of up to $15 \%$ (depending on the temperature levels of hot and cold junctions). In the proposed complex it is proposed to install Peltier elements on the surface of the evaporator in such a way that the cold junction is in contact with the surface of the evaporator heat exchanger, and the hot one is in contact with the environment (Figure 4).

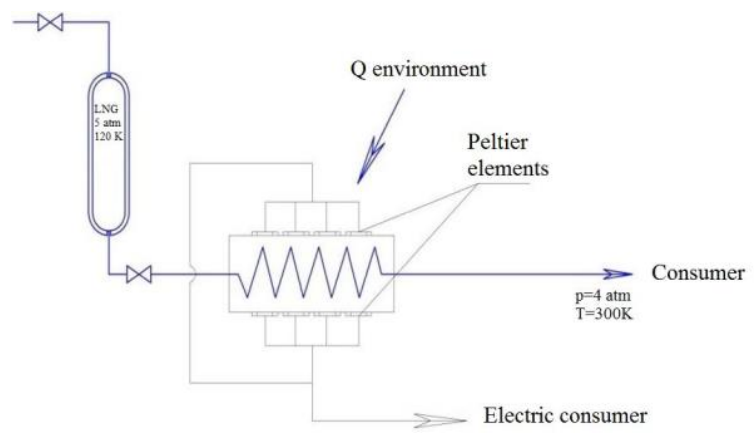

Figure 4. LNG complex with thermoelectric converters 
With a $\mathrm{LNG}$ flow rate of $\mathrm{G}=1000 \mathrm{~kg} / \mathrm{h}$ and a given efficiency, the heat flux at the cold junction $\mathrm{Q}_{\mathrm{h}}$ is about $32 \mathrm{~kW}$, and the net electrical power $\mathrm{P}=4.2 \mathrm{~kW}$ obtained, with the number of $40 \mathrm{~W}$ modules amounting to about 104 units.

\section{Conclusion}

As a result of this work, an analysis of energy losses in storage systems of cryoproducts through horizontal and vertical cylindrical tanks was carried out, energy potential losses were determined, as a result of which it was revealed that the greatest potential for cryogenic energy lost during storage is LNG. The scheme of a low-potential power plant compensating losses of a cryoproduct at its storage is analyzed.

\section{References}

1. Kakumoto, Y., Koyamatsu, Y., Shiota, A., Qudaih, Y., Mitani, Y. Application of Geographic Information System to Power Distribution System Analysis (2016) Energy Procedia, 100, pp. 360-365.

2. Burtsev, S.A., Karpenko, A.P., Leontiev, A.I. A method for distributed production of liquefied natural gas at gas-distribution stations. (2016) High Temperature, 54 (4), pp. 573-576.

3. A.M. Arkharov, I.D. Kunis. Cryogenic refueling systems of launch rocket and space complexes, publishing house MSTU. NE Bauman, 2006.

4. G. Zhang, W. Xu, Y. Yang, D. Zhang, Energy Procedia, Utilization of LNG cryogenic energy in a proposed method for inlet air cooling to improve the performance of a combined cycle, Volume 61, p. 899-903, (2014).

5. Y. Xiong, P. Luo, B. Hua, Energy Procedia, A novel CO2-capturing natural gas combined cycle with $L N G$ cold energy utilization. Volume 61, pages 899-903, (2014).

6. E.V. Blagin, A.I. Dovgyallo, S.O. Nekrasova, D.V. Sarmin, D.A. Uglanov, Applied Thermal Engineering Estimation of the energy efficiency of cryogenic filled tank using in different system and devices. 2016. Volume 101, 25 May 2016, Pages 537-544 